\title{
The increased use value of bamboo leaves as silica source for $t$-type zeolite synthesis
}

\author{
Soni Setiadji ${ }^{1, *}$, Citra Deliana Dewi Sundari ${ }^{2}$,Endang Lala $^{1}$, Denia Febby Nurbaeti $^{1}$, Ira Novianti $^{1}$, Dede Suhendar ${ }^{1}$, \\ Wahyudin Darmalaksana ${ }^{3}$, and Atthar Luqman Ivansyah ${ }^{4}$ \\ ${ }^{1}$ UIN Sunan Gunung Djati Bandung, Department of Chemistry, Faculty of Science and Technology, Bandung, Indonesia. \\ ${ }^{2}$ UIN Sunan Gunung Djati Bandung, Department of Chemistry Education, Bandung, Indonesia. \\ ${ }^{3}$ UIN Sunan Gunung Djati Bandung, Research Center, Bandung, Indonesia. \\ ${ }^{4}$ Institut Teknologi Bandung, Master Program in Computational Science, Faculty of Mathematic and Natural Science, Indonesia
}

\begin{abstract}
T-type zeolite can be used as catalyst, adsorbent, and membranes for gas separation. The synthesis of T-type zeolite needs to be optimized in both of the method and source of precursor, because of its relatively high price. In this research, the synthesis is done using silica extracted from bamboo leaves instead of commercial silica. This increases the value of the bamboo leaves and the cost-performance of zeolite synthesis. The silica was extracted from bamboo leaves ash using alkaline solvent. The extracted silica has $81.76 \%$ of purity and an amorphous phase. The T-type zeolite was synthesized using the molar composition of $0.15 \mathrm{Na}_{2} \mathrm{O}$ : $0.025 \mathrm{Al}: 0.15 \mathrm{~K}_{2} \mathrm{O}: 1 \mathrm{SiO}_{2}: 15 \mathrm{H}_{2} \mathrm{O}: 0.06 \mathrm{TMAOH}$, under hydrothermal method and heating process for 4 days, $60^{\circ} \mathrm{C}$ for 2 days and $120^{\circ} \mathrm{C}$ for the next 2 days. The characterization method using X-Ray Diffraction and Infra-Red Spectroscopy were performed to confirm the formation of T-type zeolite. The results of Scanning Electron Microscope (SEM) analysis show that the formed T-type zeolite has erionite cylindrical crystal shape.
\end{abstract}

\section{Introduction}

Zeolites are crystalline hydrated aluminosilicate minerals containing alkaline or alkaline earth cations within its three-dimensional framework. Until now, there are many known types of zeolite structure that have been successfully synthesized, and among them is T-type zeolite [1-3]. T-type zeolite is widely used for several separation processes such as the separation of $\mathrm{CO}_{2} / \mathrm{CH}_{4}$ and $\mathrm{CO}_{2} / \mathrm{N}_{2}$ mixtures [4], the separation of water/organic liquid mixtures [5], the pervaporation-aided esterification of acetic acid with ethanol [6], etc.

In general, the synthesis of zeolites uses commercial silica and aluminum sources through hydrothermal method at high temperature to produce aluminosilicate products [2]. In terms of economic value, the synthesis of zeolites using such commercial materials requires relatively high cost, so an alternative is needed to replace these commercial materials with materials that are easy to obtain and have a relatively cheaper price. The use of commercial materials especially the silica source and the inefficient method of T-type zeolite synthesis will cause the resulting T-type zeolite to have a relatively low costperformance value so that an alternative solution is needed to increase it.

Previous research has shown that silica can be extracted from several parts of plants such as rice husks, bagasse, bamboo leaves, corncobs, and peanut shells [7]. However, until now there are no researchers who use bamboo leaves waste as silica source for synthesis of Ttype zeolite. Bamboo leaves are selected as a source of

\footnotetext{
* Corresponding author: s.setiadji@uinsgd.ac.id
}

silica because it is widely available in the environment and has not been fully utilized.

In this research, the synthesis of T-type zeolite with hydrothermal synthesis method [2] has been done using bamboo leaves as silica source. It is hoped that the advantage of this study can contribute to increase the value of bamboo leaves in Indonesia and the resulting Ttype zeolite has a relatively high cost-performance value.

\section{Methodology}

The methods used in this research include silica extraction from bamboo leaves ash, T-type zeolite synthesis and its characterizations. The first stage of this research is silica extraction from the bamboo temen / awi temen (Gigantochloa atter) leaves obtained from the plantation area in Bandung, West Java, Indonesia. Cleaned bamboo leaves that had been dried indoors were collected in drums and burned to charcoal. The charcoal was then combusted in a furnace at $650^{\circ} \mathrm{C}$ for 5 hours. The resulting bamboo leaves ash was dissolved in $1 \mathrm{M}$ $\mathrm{NaOH}$ solution while heated on a hot plate at $85^{\circ} \mathrm{C}$ for 1 hour. The mixture was filtered and $3 \mathrm{M} \mathrm{H}_{2} \mathrm{SO}_{4}$ solution was added to the filtrate until white gel was formed $(\mathrm{pH}$ $\approx 7$ ). The formed white gel was set overnight for the aging process. The gel was washed using hot demineralised $(\mathrm{DM})$ water repeatedly, then filtered and dried in an oven at $110^{\circ} \mathrm{C}$ for 12 hours. The dried gel was crushed to powder and then used as a silica source for Ttype zeolite synthesis. The extracted silica from bamboo 
leaves ash was characterized using XRF (X-Ray Fluorescence), X-Ray Diffraction (XRD) and Infra-Red Spectroscopy.

T-type zeolite was hydrothermally synthesized in an autoclave using TMAOH as organic template. The materials used for the synthesis include extracted silica from bamboo leaves, aluminum tape, sodium hydroxide (98\% wt., Merck), potassium hydroxide (84\% wt., Merck), tetramethyl ammonium hydroxide (25\% wt., Merck) and DM water. The molar ratio used for this synthesis refers to the previous study [2], i.e., $0.15 \mathrm{Na}_{2} \mathrm{O}$ : $0.025 \mathrm{Al}: 0.15 \mathrm{~K}_{2} \mathrm{O}: 1 \mathrm{SiO}_{2}: 15 \mathrm{H}_{2} \mathrm{O}: 0.06$ TMAOH. The mixture was made by dissolving 1.2 grams of $\mathrm{NaOH}$ in $10 \mathrm{~mL}$ of $\mathrm{DM}$ water, and 0.135 grams of aluminum tape was added and stirred until completely dissolved, and then poured into a polypropylene bottle. Into the mixture, 1.68 grams of $\mathrm{KOH}, 17 \mathrm{~mL}$ of $\mathrm{DM}$ water and $0.54 \mathrm{~mL}$ of TMAOH were added gradually and stirred until all solids dissolved. Then 10,52 grams of silica was added, and the mixture was set covered at room temperature while stirred for 12 hours. The gel solution was transferred into an autoclave and heated for 4 days in the oven. The heating process was divided into two conditions, $60^{\circ} \mathrm{C}$ for 2 days and then $120^{\circ} \mathrm{C}$ for the next 2 days. The mixture was washed by centrifugation using DM water repeatedly, and dried at $100^{\circ} \mathrm{C}$ for 12 hours. The resulting T-type zeolite was calcined at $550^{\circ} \mathrm{C}$ for 7 hours [2]. The synthesized T-type zeolite were characterized using X-Ray Diffraction (XRD), Infra-Red Spectroscopy and Scanning Electron Microscope (SEM).

\section{Results and discussion}

The initial stage of this research is the process of silica extraction from bamboo leaves. This process is based on the principle that silica dissolves in an alkaline solution and easily precipitates when acid solution is added until it reaches neutral $\mathrm{pH}$ [7]. The reaction that occurs between $\mathrm{SiO}_{2}$ contained in bamboo leaves when dissolved in $\mathrm{NaOH}$ solution is given in equation (1):

$$
\mathrm{SiO}_{2(s)}+2 \mathrm{NaOH}_{(a q)} \rightarrow \mathrm{Na}_{2} \mathrm{SiO}_{3(a q)}+\mathrm{H}_{2} \mathrm{O}_{(l)}
$$

The reaction produces a solution of sodium silicate. The sodium silicate solution was reacted with $\mathrm{H}_{2} \mathrm{SO}_{4}$ solution until $\mathrm{SiO}_{2}$ gel was obtained. The reaction is shown in equation (2):

$\mathrm{Na}_{2} \mathrm{SiO}_{3(a q)}+\mathrm{H}_{2} \mathrm{SO}_{4(a q)} \rightarrow \mathrm{SiO}_{2(s)}+\mathrm{Na}_{2} \mathrm{SO}_{4(a q)}+\mathrm{H}_{2} \mathrm{O}_{(l)}$

The $\mathrm{H}_{2} \mathrm{SO}_{4}$ solution serves to provide an acidic condition, removing the impurities of inorganic compounds present in bamboo leaves ash (fig. 6(a)), and reacts with $\mathrm{Na}_{2} \mathrm{SiO}_{3}$ producing $\mathrm{Na}_{2} \mathrm{SO}_{4}, \mathrm{SiO}_{2}$, and water in neutral $\mathrm{pH}$ in gel form. In this process, the formation of siloxane functional groups $(\mathrm{Si}-\mathrm{O}-\mathrm{Si})$ and silanol $(\mathrm{Si}-$ $\mathrm{OH}$ ) groups occur to form silica gel. The sodium sodium salt ions formed and attached to the silica gel can be removed by washing it using the hot DM water repeatedly. The silica gel is heated in the oven at a temperature of $110^{\circ} \mathrm{C}$ resulting in dehydration of the silica gel and forming an amorphous and hard solid called dry silica gel [7]. From XRF results, the purity of extracted silica (fig. 6(b)) from bamboo leaves is about $81.76 \%$.

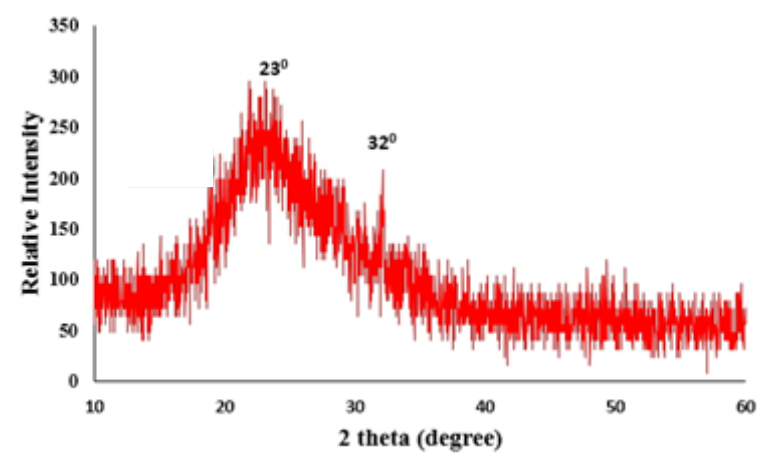

Fig. 1. XRD spectrum of the extracted silica.

Fig. 1 shows the XRD spectrum of the silica from bamboo leaves. It's concluded that the silica extracted from bamboo leaves ash has an amorphous phase, which is indicated by the presence of a broad peak at $2 \theta=$ $23^{\circ}[8]$. The amorphous phase of silica is more reactive than the crystalline phase so it is suitable as a precursor for the synthesis of zeolites. The reactivity of amorphous silica is due to the presence of silanol $(\mathrm{Si}-\mathrm{OH})$ and siloxane ( $\mathrm{Si}-\mathrm{O}-\mathrm{Si})$ groups which are the active side on the surface. However, in addition to the amorphous phase, there is a small amount of crystalline silica which is indicated by a sharp peak at $2 \theta=32^{\circ}[7,8]$. The formation of this crystalline phase may occur at the time of the drying process at $110^{\circ} \mathrm{C}$ for 12 hours or could also be affected by impurities such as potassium and sodium which can cause the decrease of melting point of silica so as to speed up the phase change to crystalline.

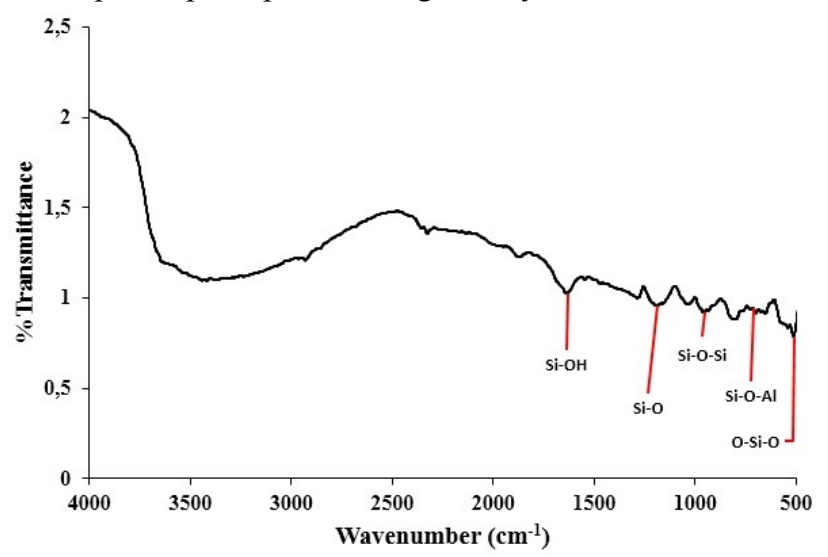

Fig. 2. IR spectrum of the extracted silica.

The identification of functional groups in the silica extracted from bamboo leaves ash can be determined by IR spectroscopy measurement and the result are given in fig. 2. The IR spectrum shows the vibration of the $-\mathrm{OH}$ functional group at the $1,651 \mathrm{~cm}^{-1}$ from $\mathrm{Si}-\mathrm{OH}$ (silanol) bonds [7]. The peak at $1,216 \mathrm{~cm}^{-1}$ is an asymmetric stretching vibration of the $\mathrm{Si}-\mathrm{O}$ bonds of the siloxane ( $\mathrm{Si}$ $\mathrm{O}-\mathrm{Si}$ ) group. The area at around $900 \mathrm{~cm}^{-1}$ shows the bonding vibration of Si-O-Si [7]. The $700 \mathrm{~cm}^{-1}$ wave number is the vibration of the Si-O-Al symmetry [7]. Then at the $500 \mathrm{~cm}^{-1}$ wave number is the bending vibration of the O-Si-O bond [7]. 
In this study, the T-type zeolite synthesis method refers to previous studies [2], but instead of using commercial silica source, the silica is extracted from bamboo leaves. The initial phase of T-type zeolite synthesis is to dissolve the aluminum tape in $\mathrm{NaOH}$ solution until completely dissolved, so that other components can react perfectly when added. Then to the aluminate solution is added solids $\mathrm{KOH}, \mathrm{TMAOH}$ and silica. The addition of $\mathrm{KOH}$ and $\mathrm{NaOH}$ other than to balance the $\mathrm{pH}$ also serves as a framework balancing cations. The hydroxide ions present in both materials serve as a good complexing for aluminates and silicates [4].

The synthesis of T-type zeolite conducted in this study using TMAOH as organic template. The addition of the organic template aims to stabilize the pore structure of T-type zeolite, so that the formation of the structure goes faster [9]. The template will form a mold and is surrounded by zeolite frame-forming ions, i.e., $\mathrm{TMA}^{+}$ cations that will react with silicate ions. After the zeolite crystals are formed, organic templates can be removed by calcination process so that the template does not affect the stability of zeolite which is usually stable in high temperature.

The process of zeolite synthesis requires different incubation time and temperature as in the synthesis of Ttype zeolite, so those two things become factors that must be taken into account so that the synthesis of zeolite becomes efficient. The method used by Yin et al. [2] become the reference in the synthesis process of this Ttype zeolite. In hydrothermal method, the temperature is one of the factors affecting the level of crystallinity of Ttype zeolite, when the temperature increases so does the level of crystallinity forming the characteristic of T-type zeolite $[3,10]$.

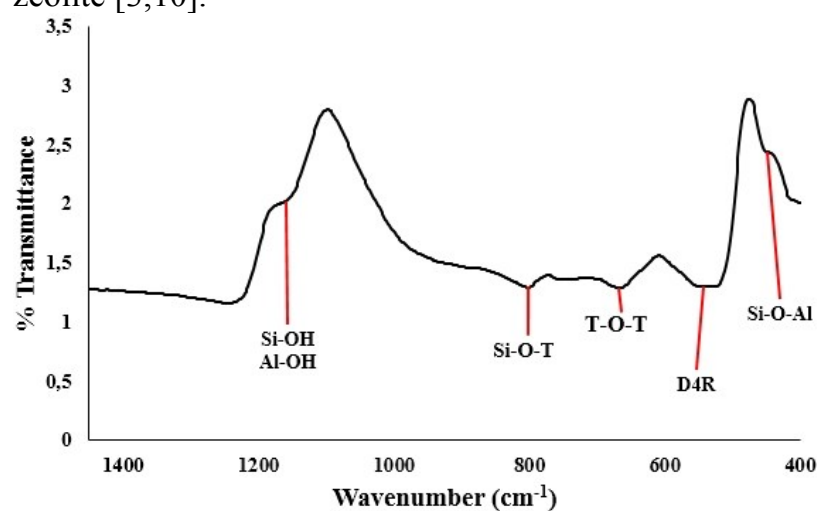

Fig. 3. IR spectrum of T-type zeolite synthesized using bamboo leaves as silica source.

The identification of functional groups in synthesized T-type zeolite (fig. 6(c)) is given in fig. 3. From the IR spectrum, T-type zeolite has an absorption band at 465 $\mathrm{cm}^{-1}$ wave number which is a bend vibration of $\mathrm{T}-\mathrm{O}$ in $\mathrm{Si}-\mathrm{O}-\mathrm{Al}$ bond which is the topology in T-type zeolite. Then the absorption band of $563 \mathrm{~cm}^{-1}$ is the secondary building unit of single ring in T-type zeolite structure and at the wave number $663 \mathrm{~cm}^{-1}$ is the $\mathrm{T}-\mathrm{O}-\mathrm{T}$ vibrational bond. Then the wavelength of $723 \mathrm{~cm}^{-1}$ is the vibration of the double ring symmetry vibration, at the wave number $800 \mathrm{~cm}^{-1}$ is $\mathrm{Si}-\mathrm{O}-\mathrm{T}$ bond and at $1,150 \mathrm{~cm}^{-1}$ asymmetry of double ring bending bands which is $\mathrm{Si}-\mathrm{O}$ and $\mathrm{Al}-\mathrm{O}$ bonds [3].

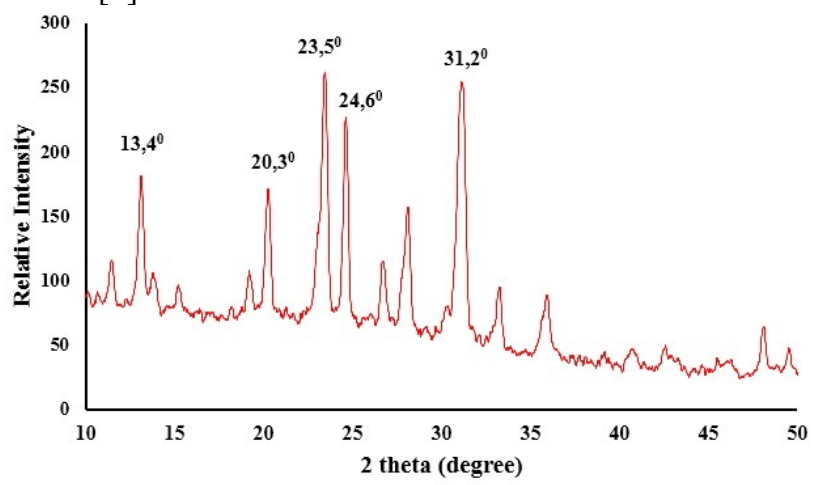

Fig. 4 XRD spectrum of T-type zeolite synthesized using bamboo leaves as silica source.

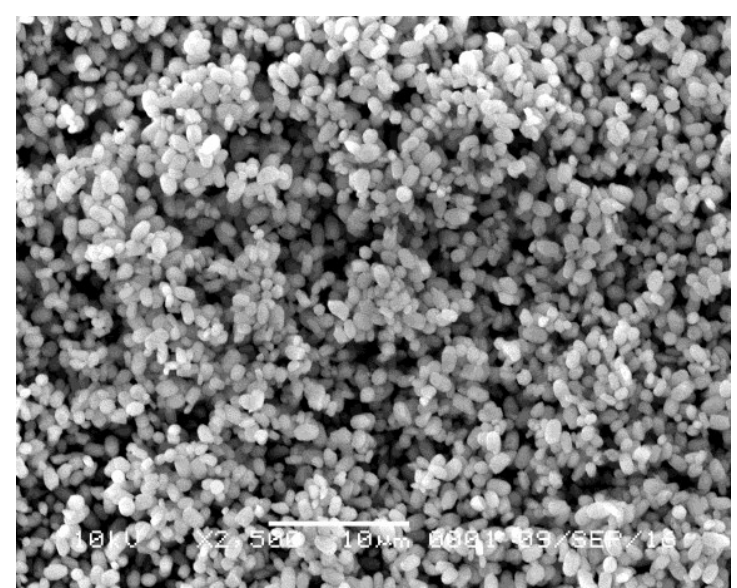

(a)

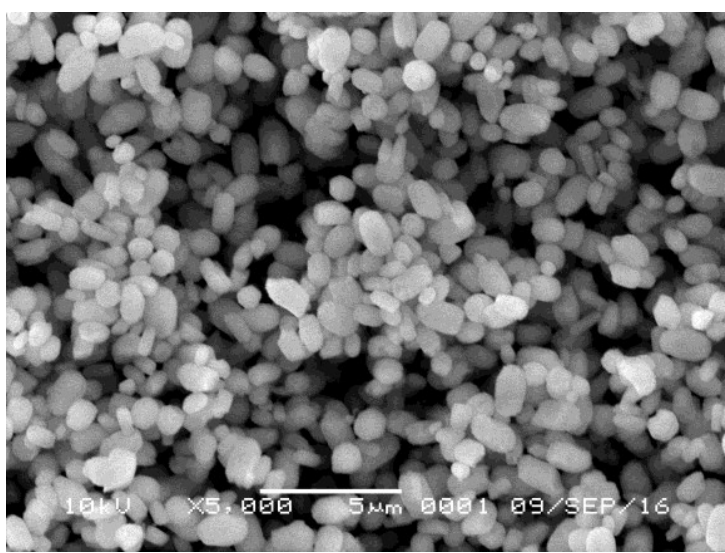

(b)

Fig. 5 SEM images of T-type zeolite synthesized using bamboo leaves as silica source, (a) $\times 2500$, (b) $\times 5000$.

From the XRD result (fig. 4.) it can be concluded that T-type zeolite can be synthesized using silica from bamboo leaves. This T-type zeolite structure gives characteristic peaks at $2 \theta=7,4^{\circ} ; 13,4^{\circ} ; 20,3^{\circ} ; 23,5^{\circ}$; $24,6^{\circ}$; and $31,2^{\circ}$. Characteristic peaks of T-type zeolite using bamboo leaves as silica source are similar with $\mathrm{T}$ type zeolite that is synthesized using commercial silica source [1-3]. The result of XRD analysis shows that the formed T-type zeolite is close to zeolite erionite type standard [3]. Erionite is a type of zeolite that is formed from hexagonal units that are not connected to six-ring plane. The T-type zeolite have a crystal size of $22.03 \mathrm{~nm}$ 
which is determined by using the Debye-Scherrer equation.

Several factors that affect the morphological shape and particle size of T-type zeolite are stirring time, incubation time, and molar composition [10]. Longer stirring time will cause the particle size to decrease. The greater the molar composition of TMAOH, the smaller the T-type zeolite shape [1].

From figure 5, it can be concluded that the particles have uniform size and cylindrical column shape [1-3]. Based on observations using the imageJ application, the particle size shown in the photo of the SEM results has the smallest particle size is $26 \mathrm{~nm}$ and $218 \mathrm{~nm}$ being the largest, with the average particle size size is $133.25 \mathrm{~nm}$.

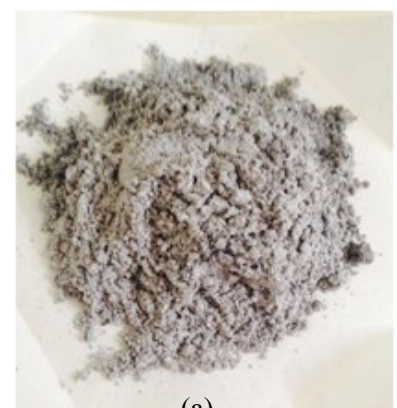

(a)
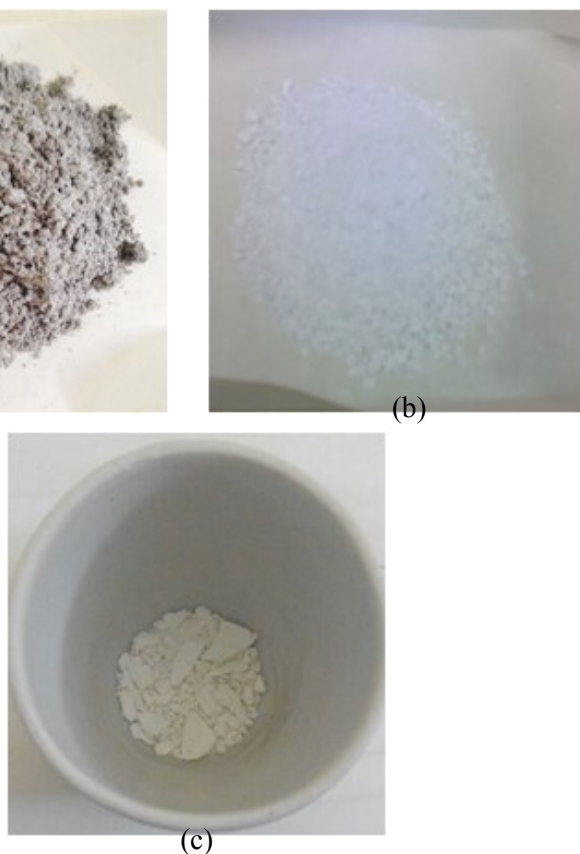

(c)

Fig. 6 (a) bamboo leaves ash, (b) silica from bamboo leaves ash and (c) T-type zeolite.

\section{Conclusion}

Bamboo leaves potentially contain silica. Silica has been successfully isolated from bamboo leaf ash using alkaline solution and has $81.76 \%$ of purity. The resulting silica has an amorphous phase and has functional group of silanol and siloxane. T-type zeolite can be synthesized using silica extracted from bamboo leaves through hydrothermal method using TMAOH. Bamboo leaves silica is a potential source of environmentally friendly silica, cheap and abundantly available for T-type zeolite synthesis. The formed T-type zeolite has a crystalline phase, cylindrical erionite type column with a crystal size of $22.03 \mathrm{~nm}$ that determined using Debye-Scherrer calculation.

This research was supported by Pusat Penelitian dan Penerbitan, Lembaga Penelitian dan Pengabdian Kepada Masyarakat (LP2M) UIN Sunan Gunung Djati Bandung.

\section{References}

1. Xiaoyan Yin, Zhongfang Li, et al., Microporous and Mesoporous Materials, 201, 247-257 (2015).

2. Xiaoyan Yin, Naibo Chu, Xuewei Lu, Zhongfang Li, Hong Guo, Solid State Sciences, 51, 30-39 (2016).

3. Mojtaba Mirfendereski, Toraj Mohammadi, Powder Technology, 206, 345-352 (2011).

4. Qiying Jiang, Jeffrey Rentschler, et al., Chemical Engineering Journal, 230, 380-388 (2013).

5. Ying Cui, Hidetoshi Kita, Ken-Ichi Okamoto, Journal of Membrane Science, 236, 17-27 (2004).

6. Kazuhiro Tanaka, Ryuuhei Yoshikawa, et al., Catalysis Today, 67, 121-125 (2001).

7. Vineet Vaibhav, U. Vijayalakshmi, S. Mohana Roopan, Spectrochimica Acta Part A: Molecular and Biomolecular Spectroscopy, 139, 515-520 (2015).

8. Jingduo Feng, Duyen Le, Son T. Nguyen, et al., Colloids and Surfaces A: Physicochemical and Engineering Aspects, 506, 298-305 (2016).

9. Korosh Shafiei, Mansour Kazemi Moghaddam, et al., Journal Particulate Science and Technology, 32, 8-19 (2014).

10. Norwahyu Jusoh, Yin Fong Yeong, et al., Ultrasonics Sonochemistry, 34, 273-280 (2017). 